\title{
GALILEO OBSERVATIONS OF ION CYCLOTRON WAVES IN THE IO TORUS
}

\author{
X. Blanco-Cano ${ }^{1}$, C. T. Russell ${ }^{2}$, R. J. Strangeway ${ }^{2}$, M. G. Kivelson ${ }^{2}$ and K. K. Khurana ${ }^{2}$ \\ ${ }^{I}$ Instituto de Geofisica, UNAM, Ciudad Universitaria, Coyoacan, D. F. 04510, Mexico \\ ${ }^{2}$ Institute of Geophysics and Planetary Physics, UCLA, Los Angeles, CA 90095-1567, USA
}

\begin{abstract}
Ion cyclotron waves generated near Io have been observed on four Galileo passes of the plasma torus, in December 1995, October 1999, November 1999, and February 2000. The waves have frequencies near the gyrofrequencies of $\mathrm{SO}_{2}^{+}$and $\mathrm{SO}^{+}$ions, and are propagating at angles up to $40^{\circ}$ to the ambient magnetic field. These waves are generated by ring-beam distributions of $\mathrm{SO}_{2}^{+}$and $\mathrm{SO}^{+}$pickup ions. In some regions there are also waves with frequencies near the gyrofrequency of $S^{+}$ions. This suggests that ring-beam sulfur ions can also make the plasma unstable. We perform a kinetic dispersion analysis for a plasma with ring-beam $\mathrm{SO}_{2}^{+}, \mathrm{SO}^{+}$, and $\mathrm{S}^{+}$pickup ions. We find that simultaneous growth of parallel propagating $\mathrm{SO}_{2}^{+}, \mathrm{SO}^{+}$, and $\mathrm{S}^{+}$cyclotron waves is possible and that the dominant mode depends on the density of the ring-beams.

(O) 2001 COSPAR. Published by Elsevier Science Ltd. All rights reserved.
\end{abstract}

\section{INTRODUCTION}

When the Galileo spacecraft flew by Io on December 7, 1995, magnetometer data revealed the existence of ion cyclotron waves in the Io torus (Kivelson et al., 1996; Huddleston et al., 1997; Warnecke et al., 1997). To a first approach these waves were propagating mainly along the magnetic field with frequencies near the $\mathrm{SO}_{2}^{+}$gyrofrequency. These waves are generated by anisotropic ring-beam $\mathrm{SO}_{2}^{+}$pickup ions. A more detailed study showed that in some regions the waves propagate at oblique angles and grow over a finite bandwidth with frequencies near the $\mathrm{SO}^{+}$gyrofrequency (Russell and Huddleston, 2000; Blanco-Cano et al., 2001a). The fact that some waves had frequencies near the $\mathrm{SO}^{+}$gyrofrequency suggests that in some regions there are $\mathrm{SO}^{+}$pickup ions able to generate cyclotron waves.

Galileo passed close to Io three more times, on October 11, 1999, on November 26, 1999 and on February 22,2000 . We study the characteristics of the observed waves within $20 R_{I o}$ of Io. We perform kinetic dispersion analysis to understand the generation of the waves, and predict maximum growth rates of ion cyclotron waves due to $\mathrm{SO}_{2}^{+}, \mathrm{SO}^{+}$, and $\mathrm{S}^{+}$ring-beam distributions.

\section{OBSERVATIONS}

Figure 1 shows examples of power spectra of the waves observed on the four days when Galileo crossed the torus. On December, 1995 (flyby I0) the waves had frequencies centered at the $\mathrm{SO}_{2}^{+}$gyrofrequency and in some intervals between the gyrofrequencies of the $\mathrm{SO}_{2}^{+}$and $\mathrm{SO}^{+}$ions. The power spectra show broad peaks, i.e., no separate peaks were observed for the $\mathrm{SO}_{2}^{+}$and $\mathrm{SO}^{+}$gyrofrequencies. Dynamic spectra (Russell and Huddleston, 2000; Blanco-Cano et al., 2001a) show that the waves are transverse, left-handed, and propagate mainly along the magnetic field. There are also regions where the angle of propagation reaches $40^{\circ}$. The waves at frequencies near the $\mathrm{SO}_{2}^{+}$gyrofrequency had larger amplitudes than at frequencies near the $\mathrm{SO}^{+}$ gyrofrequency. In contrast, on the second flyby (October 11, 1999; flyby I24), wave power spectra show two separate peaks at the $\mathrm{SO}_{2}^{+}$and $\mathrm{SO}^{+}$gyrofrequencies. The waves at frequencies near the $\mathrm{SO}^{+}$gyrofrequency were stronger and more persistent than those near the $\mathrm{SO}_{2}^{+}$gyrofrequency. They were transverse and dynamic spectra (Russell et al., 2001) show that they are left-handed with angles of propagation up to $\sim 30^{\circ}$. There were also intervals where a smaller amplitude peak appeared in the spectra near the $S^{+}$ gyrofrequency. Galileo crossed the torus for the third time on November 26, 1999. Unfortunately due to a 


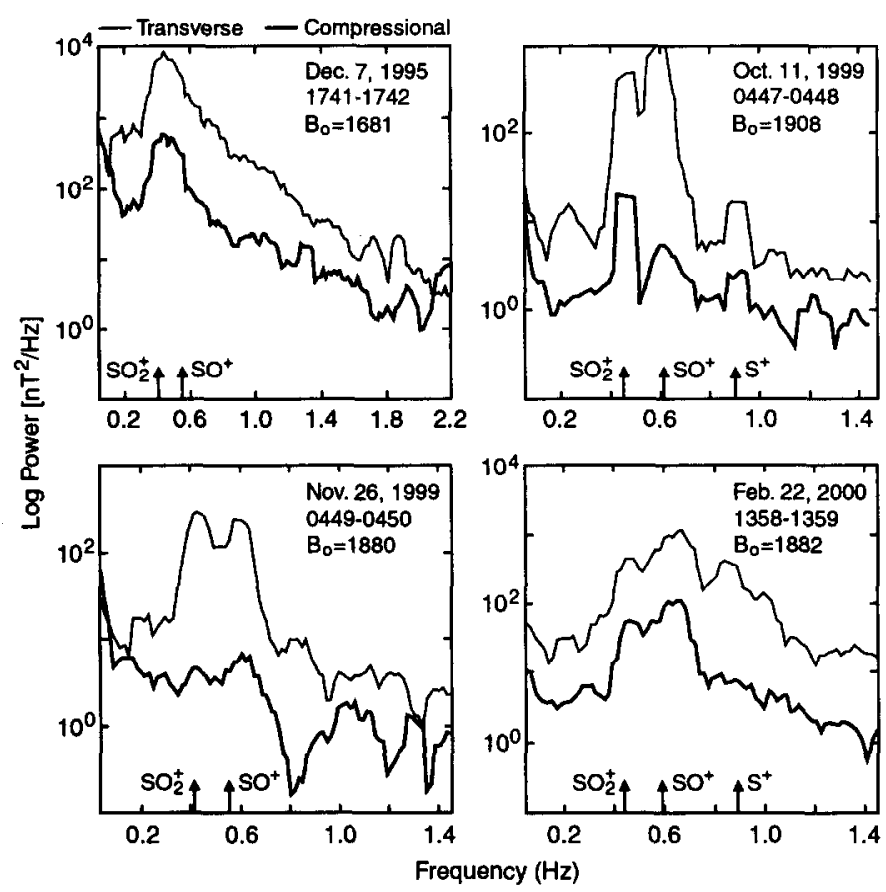

Fig. 1. Sample spectra of the waves observed by Galileo during four encounters. The thick line is the compressional power and the thin line is the transverse power. Fourier estimates of the power were added together in bands of 5 frequencies. The arrows show the heavy ion gyrofrequencies.

spacecraft safing event a few hours prior to encounter the instrument was not turned on early enough and only a short section of data after closest approach is available for this flyby. In this case the spectra show peaks with similar amplitudes near the $\mathrm{SO}_{2}^{+}$and $\mathrm{SO}^{+}$gyrofrequencies. The fourth flyby was on February 22,2000 . On this day the wave spectra show peaks near the $\mathrm{SO}_{2}^{+}$and $S \mathrm{O}^{+}$gyrofrequencies. A third peak comparable to the $\mathrm{SO}_{2}^{+}$peak is also present near the $S^{+}$gyrofrequency. In fact, this peak is more centered at the $\mathrm{H}_{2} \mathrm{~S}^{+}$gyrofrequency. The predominant waves had frequencies near the $\mathrm{SO}^{+}$gyrofrequency. The waves were transverse, left-handed and propagated at angles up to $30^{\circ}$ relative to the field.

In summary, there are differences between the waves seen on the four passes. Some of those differences may result from changes in the composition of Io's atmosphere (Russell and Kivelson, 2000), but others may be due to the different plasma torus conditions. While the December 1995 pass was at low Jupiter magnetic latitudes in a plasma with electron density $n_{e} \sim 4000 / c c$ (Gurnett et al., 1996), the October pass was at higher latitudes with $n_{e} \sim 2500 / c c$ (D. A. Gurnett and W. S. Kurth, personal communication). The composition of the torus is not well known. According to Voyager data the torus consists mainly of $\mathrm{O}^{+}$, $S^{++}$, and $S^{+}$ions (Bagenal, 1994) and few $\mathrm{SO}_{2}^{+}$pickup ions, which dissociate on time scales shorter than the corotation time. The Galileo plasma instrument (PLS) reported a similar composition in the Io wake on December, 1995 (Frank et al., 1996), with the addition of $\mathrm{SO}_{2}^{+}$estimated as $5 \%$ of the total density. Ion cyclotron waves are generated by pickup ions injected in the torus. The variability in the waves properties reveals that the torus composition is changing, and that the spatial distribution of pickup ions is not uniform.

Figure 2 shows the trajectories of Galileo through the torus. The location of waves with frequencies near the $\mathrm{SO}_{2}^{+}, \mathrm{SO}^{+}$, and $\mathrm{S}^{+}$gyrofrequencies is indicated. On December 1995, the spacecraft flew downstream from Io through the wake and $S O_{2}^{+}$cyclotron waves were observed from $20 R_{I o}$ inbound out to $7 R_{I o}$ outbound. In the edges of the wake, mirror mode waves were identified (Russell et al., 1999). In October 1999 the onset of $\mathrm{SO}^{+}$waves occurs before that of $\mathrm{SO}_{2}^{+}$waves. As Galileo moved away from Io, there are regions where a third peak near the $S^{+}$gyrofrequency was found in the spectra. At the point where the I0 and I24 trajectories crossed, the amplitude of the I24 waves integrated over both peaks was half that seen during flyby I0, $15 \mathrm{nT}$ root mean squared (rms) versus $30 \mathrm{nT}$ in 1995. For November 26, 1999 there are only a few 


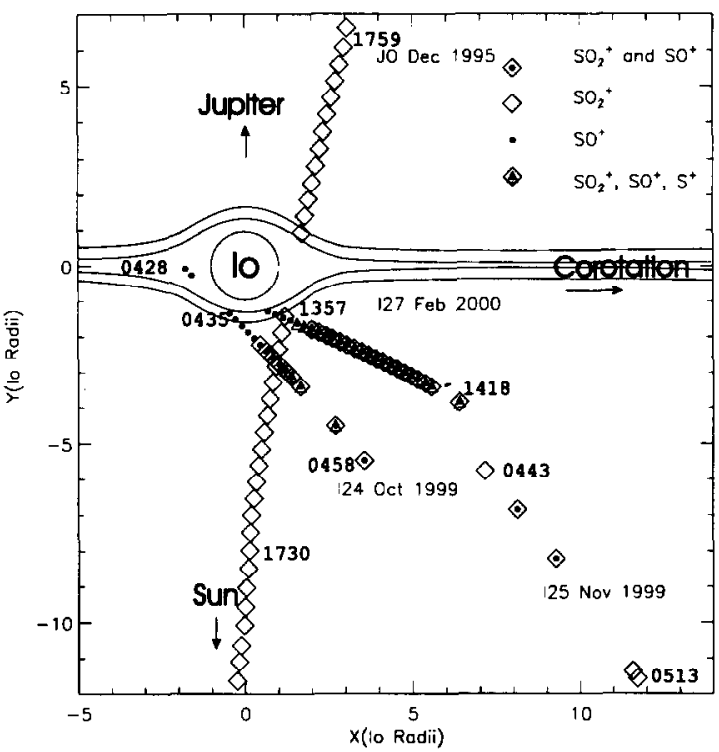

Fig. 2. The trajectories of Galileo relative to to on the encounters of December, 1995; October 1999; November 1999, and February, 2000. The locations of waves with frequencies close to the $\mathrm{SO}_{2}^{+}, \mathrm{SO}^{+}$. and $S^{+}$are indicated. Numbers along each trajectory indicate time of observation.
Table 1. Parameters for Dispersion Analysis with $\mathrm{SO}_{2}^{+}$and $\mathrm{SO}^{+}$ring-beam ions. $\mathrm{Bo}_{o}=1700 \mathrm{nT}$. $\mathrm{O}^{+}$ and $\mathrm{S}^{++}$densities are combined as a single $\mathrm{O}^{+}$component since the gyroresonance is the same for both.

\begin{tabular}{lllll} 
Species & $\mathrm{M} / \mathrm{q}$ & $\begin{array}{l}\text { Density } \\
(/ \mathrm{cc})\end{array}$ & $\begin{array}{l}T_{\|} \\
(\mathrm{eV})\end{array}$ & $\begin{array}{l}V_{i n j} \\
(\mathrm{~km} / \mathrm{s})\end{array}$ \\
\hline$S O_{2}^{+}$ & 64 & 100 & 5 & 57 \\
$S O^{+}$ & 48 & 50 & 5 & 57 \\
$O^{+}, S^{++}$ & 16 & 2800 & 100 & 0 \\
$S^{+}$ & 32 & 800 & 100 & 0 \\
$H^{+}$ & 1 & 200 & 100 & 0 \\
\hline
\end{tabular}

Table 2. Parameters for Dispersion Analysis with $\mathrm{SO}_{2}^{+}, \mathrm{SO}^{+}$, and $\mathrm{S}^{+}$ring-beam ions. $B_{o}=1900$ $\mathrm{nT}$.

\begin{tabular}{lllll} 
Species & $\mathrm{M} / \mathrm{q}$ & $\begin{array}{l}\text { Density } \\
(/ \mathrm{cc})\end{array}$ & $\begin{array}{l}T_{\|} \\
(\mathrm{eV})\end{array}$ & $\begin{array}{l}V_{\text {inj }} \\
(\mathrm{km} / \mathrm{s})\end{array}$ \\
\hline$S O_{2}^{+}$ & 64 & 25 & 5 & 57 \\
$S O^{+}$ & 48 & 125 & 5 & 57 \\
$O^{+}, S^{++}$ & 16 & 1750 & 100 & 0 \\
$S^{+}$ & 32 & 500 & 100 & 0 \\
$S^{+}($ring $)$ & 32 & 50 & 5 & 57 \\
$H^{+}$ & 1 & 125 & 100 & 0 \\
\hline
\end{tabular}

data points due to the spacecraft safing event, and these show $\mathrm{SO}_{2}^{+}$and $\mathrm{SO}^{+}$cyclotron waves. In February, 2000, Galileo entered the torus upstream from Io but detected waves only on the downstream side. The onset of $\mathrm{SO}^{+}$waves was closer to Io than that of $\mathrm{SO}_{2}^{+}$and $\mathrm{S}^{+}$waves. At the crossing point of the two trajectories, the $\mathrm{SO}_{2}^{+}$cyclotron waves observed in flyby I0 had rms amplitudes three times larger than the $\mathrm{SO}^{+}$waves of flyby $\mathrm{I} 27$ ( $75 \mathrm{nT}$ and $25 \mathrm{nT}$, respectively). The waves observed on December, 1995, suggest that at that time, $\mathrm{SO}_{2}^{+}$ions were the main component of pickup ions. In contrast, the properties of the waves on flybys I24, I25, and I27 suggest that there are regions in the torus where $\mathrm{SO}^{+}$pickup ions are dominant, and regions where ring-beam $S^{+}$ions may be able to generate waves near the $S^{+}$gyrofrequency.

The regions of cyclotron wave generation seen by Galileo and the absence of this phenomenon in the Voyager data, $10 R_{I o}$ beneath Io, constrain the dimensions of the mass-loading region where pickup ions are injected into the torus. On flyby I0 the region with waves is asymmetric in the radial direction, extending $20 R_{I o}$ in the anti-Jupiter direction and only $7 R_{I o}$ in the direction towards Jupiter. The I24 pass shows that in the upstream direction there is no wave generation beyond $\sim 2 R_{I o}$. The data of flyby I25 reveal smaller amplitude waves $(<10 n T)$ up to $12 R_{I o}$ downstream from Io. The I27 pass shows waves only downstream from Io extending as far as $6 R_{I o}$. These observations are consistent with the scenario of Russell and Kivelson (2000), where the region of the torus in the anti-Jupiter direction is favored for wave growth. The generation of the mass-loading region is explained in Russell et al. (2001, this issue).

\section{DISPERSION ANALYSIS}

To find which modes are unstable near Io we use a linear, kinetic plasma dispersion solver for waves at parallel propagation (Huddleston et al., 1997). We model the torus background distributions of $\mathrm{O}^{+}, \mathrm{S}^{+}$, $S^{++}$and $H^{+}$as Maxwellians with temperature $T_{||}=T_{\perp}=100 \mathrm{eV}$. The ring-type distributions of pickup ions have a beam perpendicular velocity, $v_{r}=V_{i n j} \sim 57 \mathrm{~km} / \mathrm{s}$, and zero drift parallel to $B_{0}, v_{b}=0$. The rings have a small thermal spread, $T_{||}=T_{\perp}=5 \mathrm{eV}$. Fluctuations are considered small enough to justify a linear approximation. In the torus the high magnetic field strength ensures $\delta B / B$ is small and we should have an approximately linear regime. Electrons have $n_{e}=\sum_{i} n_{i}$, and are assumed to be cold, with $v_{T \|}=v_{T \perp}=0$. 


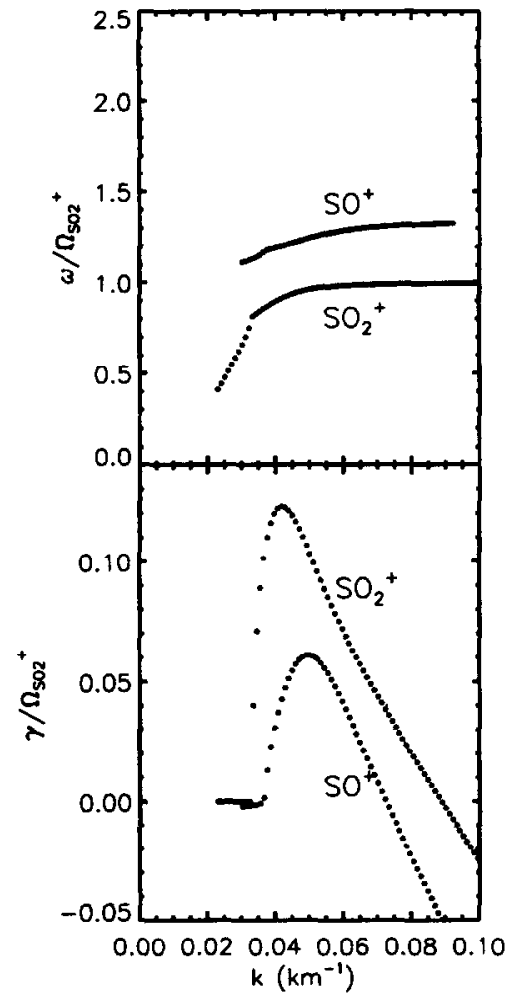

Fig. 3. Dispersion relation of parallel propagating cyclotron waves generated by $\mathrm{SO}_{2}^{+}$and $\mathrm{SO}^{+}$ringbeam ions. $n_{\mathrm{SO}_{2}^{+}}=100 / \mathrm{cc}$ and $n_{\mathrm{SO}^{+}}=50 / \mathrm{cc}$ (see Table 1). $\omega$ is the frequency, $\gamma$ is the growth rate, and $k$ is the wave number. $\gamma>0$ corresponds to growing waves. $\Omega_{\mathrm{SO}_{2}^{+}}$is the gyrofrequency of $\mathrm{SO}_{2}^{+}$ions.

\section{December 7, 1995}

Figure 3 shows the dispersion relation of the waves that are unstable in a plasma resembling the torus on December 7, 1995 (see Table 1). The plasma has $\mathrm{SO}_{2}^{+}$and $\mathrm{SO}^{+}$pickup ions. The panels show the frequency $(\omega)$, and the growth rate $(\gamma)$ as a function of wave number (k) for the $\mathrm{SO}_{2}^{+}$and $\mathrm{SO}^{+}$parallel propagating ion cyclotron waves that are generated when the densities of $\mathrm{SO}_{2}^{+}$and $\mathrm{SO}^{+}$pickup ions are $\sim 2 \%$ and $\sim 1 \%$ the total density. The $\mathrm{SO}_{2}^{+}$ density is between the reported values (Frank et al., 1996; Huddleston et al., 1998). The density of $\mathrm{SO}^{+}$pickup ions has not been reported for this flyby but we include this component because some of the observed waves had frequencies near the gyrofrequency of $\mathrm{SO}^{+}$suggesting that $\mathrm{SO}^{+}$pickup ions are also present. The branch with $\omega \leq \Omega_{S O_{2}^{+}}$ corresponds to the wave generated by $\mathrm{SO}_{2}^{+}$ringbeam ions and the branch with $\omega>\Omega_{\mathrm{SO}_{2}^{+}}$to $\mathrm{SO}^{+}$ ion cyclotron waves. The $\mathrm{SO}_{2}^{+}$cyclotron waves have larger growth rate than the $\mathrm{SO}^{+}$waves and this is consistent with observations on December 7 , 1995 , when the $\mathrm{SO}_{2}^{+}$peak in the spectra was larger than the power of the waves with frequencies near the $\mathrm{SO}^{+}$gyrofrequency. The waves have maximum growth at wavelengths $\sim 148$ and $\sim 126 \mathrm{~km}$. The growth of $\mathrm{SO}^{+}$cyclotron waves occurs in one of the frequency bands observed in some intervals on December 7, 1995, between the $\mathrm{SO}_{2}^{+}$and the $\mathrm{SO}^{+}$ gyrofrequencies.

On December 7, 1995 most of the waves propagated at small angles to the ambient field, but there are intervals where the angle of propagation is $\sim 30-40^{\circ}$. Ion cyclotron waves have largest growth at parallel propagation. However, due to the temperature anisotropy the growth at oblique angles can be significant (Blanco-Cano et al., 2001a). In another work (Blanco-Cano et al., 2001b) we estimated the growth of $\mathrm{SO}_{2}^{+}$and $\mathrm{SO}^{+}$ion cyclotron waves at oblique angles using the dispersion solver WHAMP developed by $\mathrm{K}$. Rönmark. In this case each species was described with bi-Maxwellian $\left(T_{\perp}>T_{\|}\right)$distributions. We find that the maximum growth rate of $\mathrm{SO}_{2}^{+}$cyclotron waves propagating at $40^{\circ}$ is $\geq 70 \%$ the maximum growth rate at parallel propagation (see Figure 5 in Blanco-Cano et al., 2001b). This is consistent with observations which show that although most of the waves propagate at small angles to the magnetic field, there are regions where the angle of propagation reaches $30-40^{\circ}$. The observability of waves does not only depend on the growth rate, but also on the time that the waves spend in an unstable region. In the torus, the flow is corotating perpendicular to the magnetic field. The wave velocity is comparable to the flow velocity $(\sim 57$ $\mathrm{km} / \mathrm{s}$ ) and oblique propagating waves may stay in an unstable region longer than parallel propagating waves and this can help to increase the net growth of oblique waves.

\section{October 11, 1999, and February 22, 2000}

On October 11, 1999, and February 22, 2000 there are regions where waves with frequencies near the $\mathrm{SO}^{+}$ gyrofrequency are dominant. During these days there were also regions where a peak in the spectra, near the $S^{+}$ion gyrofrequency was observed. The PLS Galileo instrument showed the existence of $\mathrm{SO}^{+}$and $\mathrm{S}^{+}$ pickup ions during flyby I24 (Paterson and Frank, 2000). 


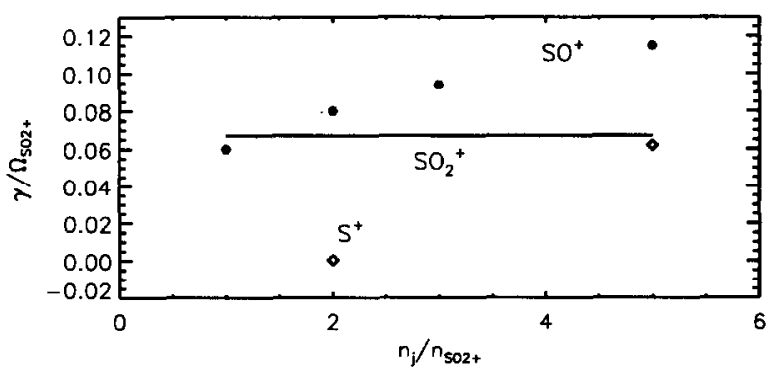

Fig. 4. Growth rate of parallel propagating cyclotron waves generated by $\mathrm{SO}_{2}^{+}$(solid line), $\mathrm{SO}^{+}$(dots), and $S^{+}$(diamonds) pickup ions. The $\mathrm{SO}_{2}^{+}$density is $25 / \mathrm{cc}$, but $n_{S O+}$ and $n_{S+\text { ring }}$ changed $\left(n_{j} / n_{S O_{2}^{+}}\right)$.
To know under which conditions the $\mathrm{SO}^{+}$cyclotron mode can be dominant, we calculate the growth rate of $\mathrm{SO}_{2}^{+}$and $\mathrm{SO}^{+}$parallel propagating cyclotron waves for different values of $n_{\mathrm{SO}^{+}} / n_{\mathrm{SO}_{2}^{+}}$. To study when sulfur cyclotron waves become unstable we included a $S^{+}$ring-beam and vary $n_{S^{+}} / n_{S O_{2}^{+}}$. Ring-beam $\mathrm{SO}^{+}, \mathrm{SO}_{2}^{+}$, and $\mathrm{S}^{+}$distributions are injected at $V_{i n j}=57 \mathrm{~km}$, with $T_{\|}=T_{\perp}=5 \mathrm{eV}$. There are no $S O_{2}^{+}$and $S O^{+}$background ions, but there is a $\mathrm{S}^{+}$background distribution (see Table 2). Figure 4 shows the maximum growth rate of the $\mathrm{SO}_{2}^{+}$and $\mathrm{SO}^{+}$ion cyclotron waves at parallel propagation.

The $\mathrm{SO}^{+}$waves have larger growth rate than the $\mathrm{SO}_{2}^{+}$waves if $n_{\mathrm{SO}^{+}} / n_{\mathrm{SO}_{2}^{+}}>2$. According to this, in the regions of the torus where Galileo observed waves on flyby I24, and during most of flyby I27, the $\mathrm{SO}^{+}$pickup ion density was at least twice the $\mathrm{SO}_{2}^{+}$density. The existence of sulfur cyclotron waves on flyby I24 and I27 came as a surprise because background $S^{+}$thermalized ions are always present in the torus and this distribution can quench the growth of a sulfur mode. However, as shown in Figures 4 and 5 , waves with frequency near the gyrofrequency of $S^{+}$ions can become unstable $\left(\gamma / \Omega_{S O 2+}=0.0004\right)$ due to a sulfur ring-beam distribution with density $n_{S^{+}} / n_{S_{2}^{+}} \geq 2\left(\geq 10 \%\right.$, the density of the $S^{+}$background ions). Increasing the density of the sulfur ring-beam to $n_{S^{+}} / n_{S_{2}^{+}}=5\left(25 \%\right.$, the background $S^{+}$density) results in $S^{+}$cyclotron waves with growth rate comparable to the growth rate of $S_{2}^{+}$waves.

Figure 5 shows the dispersion relation of the cyclotron waves that are generated in a plasma with $\mathrm{SO}_{2}^{+}$, $S \mathrm{O}^{+}$, and $S^{+}$pickup ions. We take $n_{\mathrm{SO}^{+}} / n_{\mathrm{SO}_{2}^{+}}=5$, and $n_{\mathrm{SO}_{2}^{+}}=25 / \mathrm{cc}$ (see Table 2). In agreement with observations on flyby I24 and with most of the waves on I27 the growth of $\mathrm{SO}^{+}$cyclotron waves is larger than the growth of $\mathrm{SO}_{2}^{+}$waves. The $\mathrm{S}^{+}$cyclotron wave has a growth rate similar to the growth of $\mathrm{SO}_{2}^{+}$ waves, and this is consistent with flyby I27 during which there are regions where waves with frequency near the $S^{+}$gyrofrequency had similar amplitudes to the $\mathrm{SO}_{2}^{+}$cyclotron waves. Thus, in these regions very dense $S^{+}$ring-beams must be present. On flyby I24 the peak near the $S^{+}$gyrofrequency had smaller amplitudes than the peak near the $S O_{2}^{+}$gyrofrequency, and we expect the $S^{+}$ring-beam density to be smaller. From our dispersion results we conclude that while on December 7, 1995 the major component of the pickup ions was sulfur dioxide, on October 11, 1999, and February 22, 2000, the composition of the pickup ions was different with $n_{\mathrm{SO}+} / n_{\mathrm{SO} 2+} \geq 2$ in most of the regions observed by Galileo.

On October 11, 1999 the waves propagated at angles up to $\sim 30^{\circ}$. The $\mathrm{SO}^{+}$waves were propagating initially at $\sim 20^{\circ}$ but eventually the angle of propagation dropped to $\sim 5^{\circ}$. The $\mathrm{SO}_{2}^{+}$waves propagated with angles $\geq 20^{\circ}$ all the time. Figure 8 of Blanco-Cano et al. (2001b) shows the growth rate of oblique propagating $\mathrm{SO}^{+}$and $\mathrm{SO}_{2}^{+}$waves for conditions resembling the torus on flybys I24 and I27. The growth at oblique angles is significant, with $\gamma_{\max } \geq 0.90 \gamma_{\max \|}$ for angles up to $25^{\circ}$. The normalized $\mathrm{SO}_{2}^{+}$and $\mathrm{SO}^{+}$growth rates are very similar for the two branches for angles up to $30^{\circ}$. Therefore, some nonlinear mechanism must be in operation, leading to the observed differences in the angles of propagation of the two frequency bands.

\section{DISCUSSION AND CONCLUSIONS}

Galileo data taken during four passes of the Io torus show the existence of waves with frequencies near the $\mathrm{SO}_{2}^{+}, \mathrm{SO}^{+}, \mathrm{S}^{+}$, and probably $\mathrm{H}_{2} \mathrm{~S}^{+}$gyrofrequencies. The waves are found in localized regions in the torus and their properties are not uniform. While on December, 1999 the predominant waves have frequencies near the $\mathrm{SO}_{2}^{+}$gyrofrequency, wave observations on October, 1999, and February 2000 show regions where the predominant mode has a frequency near the $\mathrm{SO}^{+}$gyrofrequency.

Dispersion analysis shows that the waves are generated by ring-beam pickup ions. It is possible to have simultaneous growth of $\mathrm{SO}_{2}^{+}, \mathrm{SO}^{+}$, and $\mathrm{S}^{+}$cyclotron waves in a plasma such as the Io torus. The dominant 


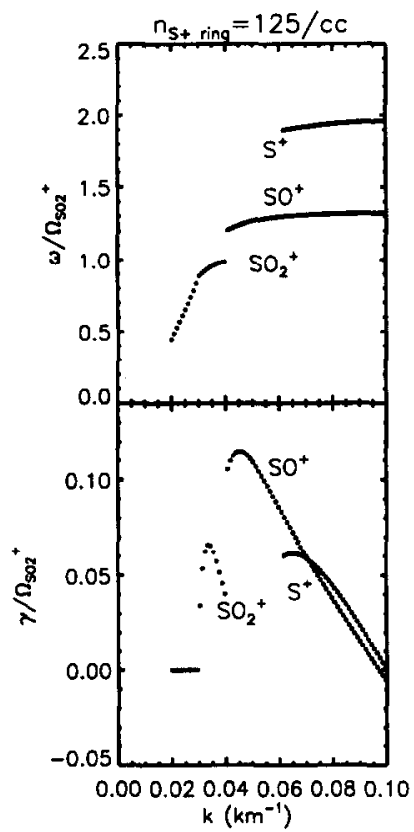

Fig. 5. $\mathrm{SO}_{2}^{+}, \mathrm{SO}^{+}$, and $\mathrm{S}^{+}$ion cyclotron waves. See Table 2, and caption of Figure 3. mode depends on the composition of the pickup ions in the plasma. We find that $\mathrm{SO}^{+}$cyclotron waves can have larger growth rate than the waves generated by $\mathrm{SO}_{2}^{+}$pickup ions if $n_{\mathrm{SO}^{+}} / n_{\mathrm{SO}_{2}^{+}} \geq 2$. Thus, according to these result in the regions of the Io torus where Galileo observed waves during flyby $\mathrm{I} 0$, the $\mathrm{SO}^{+}$pickup ions had density $n_{\mathrm{SO}+}<2 n_{\mathrm{SO} 2+}$. On flyby I24, and during most of the time of flyby $\mathrm{I} 27$, the $\mathrm{SO}^{+}$pickup ions were at least twice denser than the $\mathrm{SO}_{2}^{+}$component. $\mathrm{S}^{+}$ cyclotron waves can be unstable in the plasma torus when the $S^{+}$ring-beam density is $\geq 10 \%$ the density of the $S^{+}$background ions. Thus, in the regions where Galileo observed a peak near the sulfur gyrofrequency, dense $S^{+}$ring-beams must be present in the plasma. Part of our future work is to model the pickup ion components as ring-beam distributions to find growth rates for the $\mathrm{SO}_{2}^{+}, \mathrm{SO}^{+}$, and $S^{+}$cyclotron waves at oblique propagation.

\section{ACKNOWLEDGMENTS}

We thank the Galileo magnetometer team. XBC is supported by CONACyT.

\section{REFERENCES}

Bagenal F., Empirical model of the Io plasma torus: Voyager measurements, J. Geophys. Res., 99, 11043, 1994.

Blanco-Cano, X., Russell, C. T., Huddleston D. E., and R. J. Strangeway, Ion Cyclotron Waves Near Io, Planet. Space Sci., 49, 1125, 2001a.

Blanco-Cano, X., Russell, C. T., and R. J. Strangeway, The Io mass loading disk: wave dispersion analysis, $J$. Geophys. Res., in press, 2001b.

Frank, L. A., W. R. Paterson, K. L. Ackerson, V. M. Vasyliunas, F. V. Coroniti, and S. J. Bolton, Plasma observations at Io with the Galileo spacecraft, Science, 274, 394, 1996.

Gurnett D. A., W. S. Kurth, A. Roux, S. J. Boltonm and C. F. Kennel, Galileo plasma wave observations in the Io plasma torus and near Io, Science, 274, 391, 1996

Huddleston, D. E., R. J. Strangeway, J. Warnecke, C. T. Russell, M. G. Kivelson, and F. Bagenal, Ion cyclotron waves in the Io torus during the Galileo encounter: Warm plasma dispersion analysis, Geophys. Res. Lett. 24, (17), 2143, 1997.

Huddleston, D. E., R., J. Strangeway, J. Warnecke, C. T. Russell, and M. Kivelson, Ion cyclotron waves in the Io torus: Wave dispersion, free energy analysis, and $\mathrm{SO}_{2}^{+}$source rate estimates, J. Geophys. Res., 103, 19887, 1998.

Kivelson, M. G., K. K. Khurana, R. J. Walker, J. Warnecke, C. T. Russell, J. A. Linker, D. J., Southwood, and C. Polanskey, Io's interaction with the plasma torus: Galileo magnetometer report, Science, 234, 396, 1996.

Paterson, W. R., and Frank, L. A., Galileo PLS Observations of the Io plasma torus, AGU Spring meeting, EOS Transactions, 2000.

Russell, C. T., D. E. Huddleston, R. J. Strangeway, X. Blanco-Cano, M. G. Kivelson, K. K. Khurana, L. A. Frank, W. Paterson, D. A. Gurnett, and W. S. Kurth, Mirror-mode structures at the Galileo-Io flyby: observations, J. Geophys. Res., 104, 17471, 1999.

Russell, C. T., and D. E. Huddleston, Ion cyclotron waves at Io, Adv. Space Res.,26(10),1505, 2000.

Russell, C. T., and M. G. Kivelson, Detection of SO in Ios Exosphere, Science, 287, 1998, 2000.

Russell C. T., Y. L. Wang, X. Blanco-Cano, and R. J. Strangeway, The Io mass loading disk: constraints provided by ion cyclotron wave observations, J. Geophys. Res., in press, 2001. 